\title{
Nurses' practice concerning sedation during weaning from mechanical ventilation
}

\author{
M Borkowska ${ }^{1 *}$, S Labeau², S Blot ${ }^{1}$ \\ From ESICM LIVES 2015 \\ Berlin, Germany. 3-7 October 2015
}

\section{Introduction}

Sedation management during weaning from mechanical ventilation is an important task for ICU nurses. Nurse-led sedation protocols have a favorable impact on weaning outcome $[1,2]$.

\section{Objectives}

To identify sedation practices among ICU nurses and to determine barriers towards use of sedation protocols.

\section{Methods}

A cross-sectional, self-administrated survey was developed. Consensus on content validity was achieved through a Delphi procedure among experts. The survey was distributed and collected during the annual congress of the Flemish Society of Critical Care Nurses (Dec. 2014).

\section{Results}

423 attendants completed the survey (response rate: $66 \%$ ) of which 342 ICU nurses were included for further analysis. These are employed in general $(73 \%)$ or university hospitals $(27 \%)$ and are working in mixed $(66 \%)$, surgical (18\%) or medical (13\%) ICUs.

Short working agents are most frequently chosen for analgo-sedation. A majority of respondents administer sedatives in continuous infusion with bolus doses (81\%). Less than half of nurses have a sedation protocol (44\%) but only minority consistently uses it (8\%). Nurses in university hospitals reported higher availability of sedation protocols compared to general hospitals $(72 \%$ vs. $42 \%, \mathrm{p}<0,001)$. Patient-targeted protocols are most available (53\%). They are mostly developed particularly by MDs (79\%) and nurses (52\%). Level of sedation is generally evaluated per 2 hrs (56\%) and with use of a
RASS scale (59\%). Daily interruption of sedation (DIS) is applied variably (never: $27 \%$, rarely: $54 \%$, mostly $14 \%$, always: $2 \%$ ) and usually to evaluate the neurological status $(86 \%)$ and to shorten the duration of ventilation (44\%). $78 \%$ of nurses report not to apply DIS at night. Patients' discomfort is the most important barrier to execute DIS (49\%). Nearly half of respondents pointed out respiratory deterioration is the greatest concern during DIS (47\%). For $40 \%$ of nurses it is risk of selfextubation and removal of the catheters. Agitation/confusion (66\%) and lack of comfort and pain (46\%) are the most frequently reported problems associated with sedation reduction.

\section{Conclusions}

There appears to be a considerable discrepancy between recommendations and sedation practice. This data demonstrate room for quality improvement initiatives.

\section{Authors' details \\ ${ }^{1}$ Ghent University, Ghent, Belgium. ' University College Ghent, Ghent, Belgium.}

Published: 1 October 2015

\section{References}

1. Arias-Rivera S, et al: Crit Care Med 2008.

2. McGrane S, Pandharipande PP: Minerva Anestesiol 2012.

doi:10.1186/2197-425X-3-S1-A925

Cite this article as: Borkowska et al:: Nurses' practice concerning

sedation during weaning from mechanical ventilation. Intensive Care

Medicine Experimental 2015 3(Suppl 1):A925. 